\title{
Quasi Exact Solution of the Fisher Equation
}

\author{
G. Dattoli, E. Di Palma, E. Sabia, S. Licciardi \\ ENEA-Centro Ricerche Frascati, Via E. Fermi, Frascati, Italy \\ Email: giuseppe.dattoli@enea.it, emanuele.dipalma@enea.it, elio.sabia@enea.it, silviakant@gmail.com
}

Received April 24, 2013; revised May 24, 2013; accepted June 1, 2013

Copyright (C) 2013 G. Dattoli et al. This is an open access article distributed under the Creative Commons Attribution License, which permits unrestricted use, distribution, and reproduction in any medium, provided the original work is properly cited.

\begin{abstract}
We propose an accurate non numerical solution of the Fisher Equation (FE), capable of reproducing the known analytical solutions and those obtained from a numerical analysis. The form we propose is based on educated guesses concerning the possibility of merging diffusive and logistic behavior into a single formula.
\end{abstract}

Keywords: Non-Linear Diffusion Equation; Operational Calculus

\section{Introduction}

In this paper, we propose a "quasi-exact" solution of the Fisher equation and compare it with solutions available in the literature. We also develop an extensive numerical analysis along with an accurate comparison with the solution presented here.

The Fisher Equation (FE) introduced to describe the spreading of genes [1] has found applications in different fields of research ranging from ecology [2] to plasma physics [3]. In this paper we do not consider its applicative aspects, but we will discuss a solution of "quasi exact" nature, potentially useful for practical purposes ${ }^{1}$. The FE is a non-linear diffusion equation, which in its original formulation writes

$$
\begin{aligned}
\partial_{t} F & =D \partial_{x}^{2} F+r\left(1-\frac{F}{K}\right) F, \\
\left.F\right|_{t=0} & =g(x)
\end{aligned}
$$

In the above equation $F$ is a function of the spatial coordinate and time and the initial condition is given by $g(x)$, supposed to be a continuous infinitely differentiable function. From the dynamical point of view the equation describes a spatial diffusion in a homogeneous medium (the coefficient $D$ is assumed to be independent of the spatial coordinate) embedded with a growth of logistic nature [4].The coefficients $r$ and $K$ are the growth rate and the carrying capacity of the environment respectively [4]. If the process is purely diffusive $(r=0)$, the solution of our evolution problem is just provided by the follow-

\footnotetext{
${ }^{1}$ In view of its various applications the equation is referred also with other names (Kolmogorov, Burgers...) in this paper we avoid multiple nomenclature by just referring as FE.
}

ing Gauss Weierstrass transform [5]

$$
\tilde{F}(x, t)=\frac{1}{2 \sqrt{\pi D t}} \int_{-\infty}^{\infty} \mathrm{e}^{-\frac{(x-\sigma)^{2}}{4 D t}} g(\sigma) \mathrm{d} \sigma
$$

with $g(x)$ such that the integral in Equation (1.2) converges.

$$
\begin{aligned}
\text { In the case of } & g(x)=\mathrm{e}^{-x^{2}} \text { reduces to } \\
& \tilde{F}(x, t)=\frac{1}{\sqrt{1+4 D t}} \mathrm{e}^{-\frac{x^{2}}{1+4 D t}}
\end{aligned}
$$

In absence of the diffusion, $(D=0)$, Equation (1.1) describes a purely logistic process ${ }^{2}$ and its solution reads [4]

$$
\Phi=g_{0} \frac{\mathrm{e}^{r t}}{1+\frac{g_{0}}{K}\left(\mathrm{e}^{r t}-1\right)}
$$

Where $g_{0}$ is the initial condition associated with Equation (1.1) and is characterized by an initial exponential growth successively undergoing a saturation, when the $F$ values approaching the carrying capacity. We can provide a tentative solution for the general case, by merging the characteristics implicit in Equations (1.3) and (1.4) through the following expression

$$
F(x, t)=\frac{\mathrm{e}^{r t}}{1+\frac{1}{K}\left(\mathrm{e}^{r t} \tilde{F}(x, t)-g(x)\right)} \tilde{F}(x, t)
$$

possessing both features of spreading and logistic satura-

$$
\begin{aligned}
& { }^{2} \text { We refer to the solution of the equation } \\
& \qquad \mathrm{d} \Phi / \mathrm{d} t=r(1-\Phi / K) \Phi,\left.\Phi\right|_{t=0}=g_{0}
\end{aligned}
$$


tion. The above solution and the relevant derivation may sound as the consequences of a naive procedure without any rigor. We will provide a more sounded justification in the concluding section using an appropriate Hopf-Cole transform, here we will follow a pragmatic approach and accept it on the basis of its agreement with already exact known solutions and with full numerical solutions. Examples of evolution at different times for an initially Gaussian distribution are given in Figure 1.

The distribution has been normalized at the value taken at $x=0$ at different times and the growth of the amplitude at the origin and for $x=7$ is shown in Figure 2, the behavior is that of a logistic curve with the same carrying capacity and different initial conditions.

As we will see in the forthcoming section, the process described by Equation (1.1) can be viewed as a kind of travelling wave front at a given velocity, within the frame-

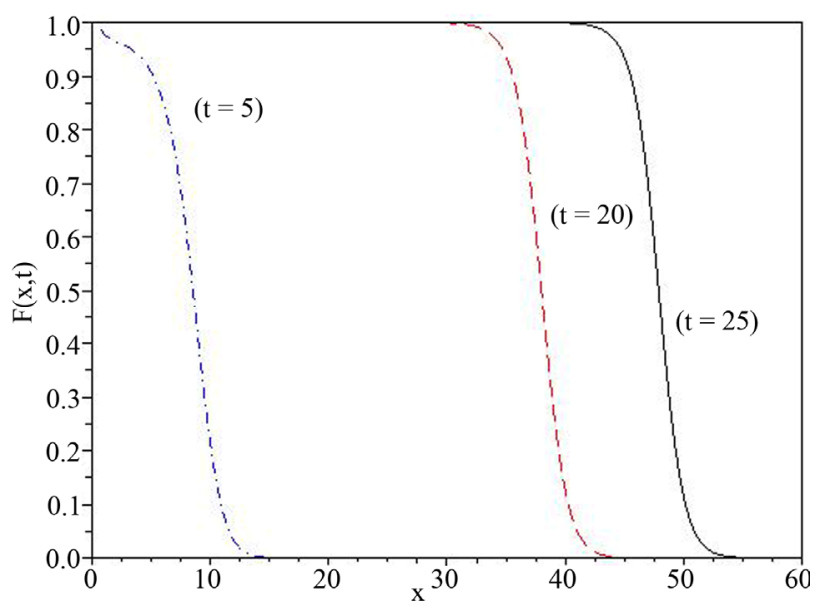

Figure 1. Evolution of an initially Gaussian distribution $\left(g(x)=\exp \left(-x^{2}\right)\right)$ for $D=1, r=1, K=1$ at different times $t=5$ (dash), $t=20$ (dot) $t=25$ (continuous).

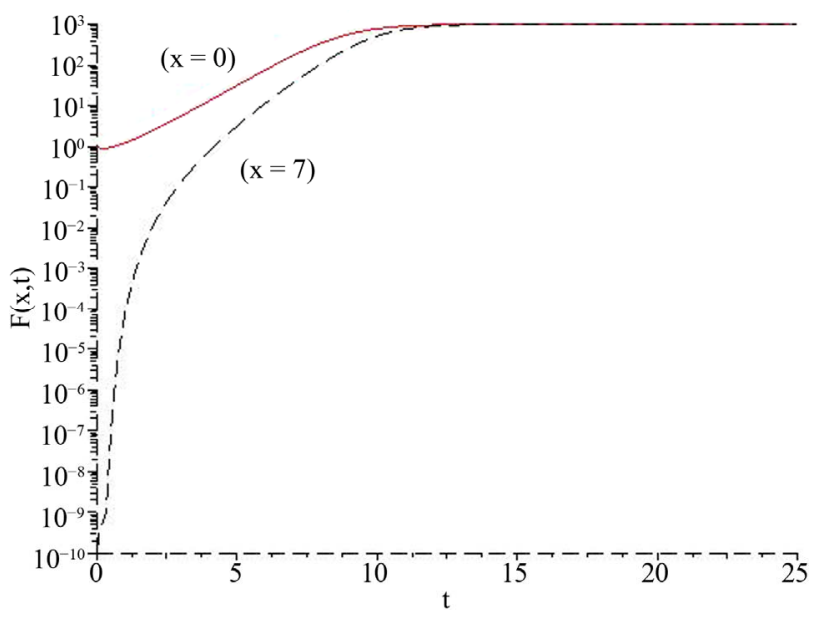

Figure 2. Evolution vs. time of the function amplitude for two different points $(x=0$ continuous and $x=7$ dot) same parameters of Figure $1\left(K=10^{3}\right)$. work of the previous solution the front velocity can be evaluated as

$$
\begin{aligned}
& V_{f}=\frac{\mathrm{d}}{\mathrm{d} t} \bar{X}(t), \bar{X}(t)=\int_{0}^{\infty} x \Psi(x, t) \mathrm{d} x, \\
& \Psi(x, t)=\frac{F(x, t)}{\int_{0}^{\infty} F(x, t) \mathrm{d} t}
\end{aligned}
$$

The behavior of the velocity vs. the time is reported in Figure 3 for different values of the parameters. Our results allow to confirm that (see [6]) the asymptotic velocity (namely the long time velocity) scales as

$$
\left.V_{f}\right|_{t \rightarrow \infty} \cong \sqrt{r D}
$$

while for short "times"

$$
V_{f} \propto \frac{1}{t} \ln (t)
$$

We have so far shown that our guessed solution is compatible with the phenomenology of the FE, in the forthcoming sections we will compare it with known analytical solutions and with numerical solutions obtained with an ad hoc developed code.

\section{Comparison with Analytical Solutions}

Various forms of exact solutions for the Fisher equation have been provided in the past. They are limited to specific cases of the initial function and/or of the constant entering the equation itself. Albeit limited to specific cases these solution provides an important test for comparison with our quasi exact form. One of these solutions, proposed in [7-9], is given by

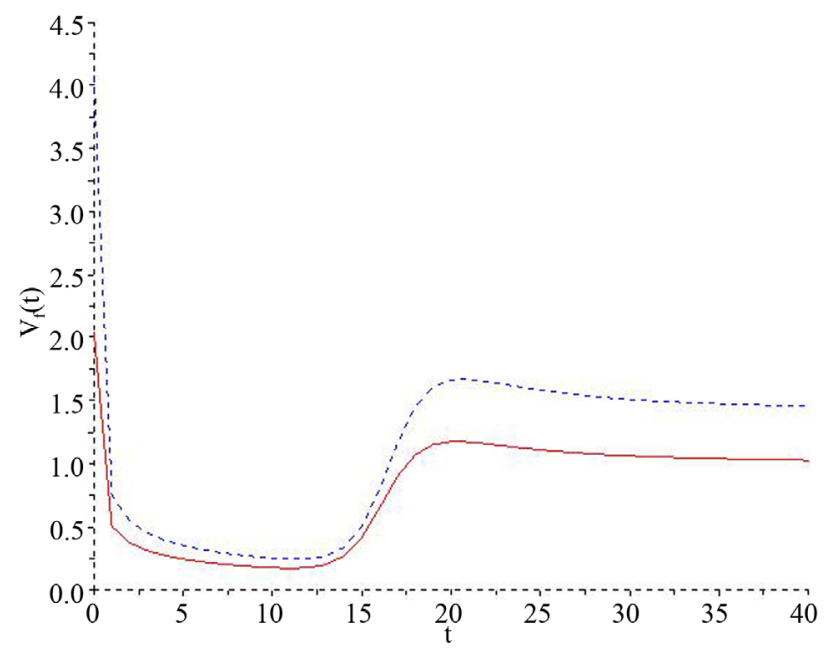

Figure 3. Front wave velocity vs. time $\left(r=1, D=1, K=10^{3}\right.$ continuous line, $r=1, D=2, K=10^{3}$ dot line). 


$$
G(x, t)=\frac{1}{\left[1+C \cdot \mathrm{e}^{-\frac{5}{6} \cdot r \cdot t+\frac{\sqrt{6 r}}{6} x}\right]^{2}}
$$

and $C$ is an arbitrary constant. To make a comparison we use Equation (1.5) with

$$
g(x)=\frac{1}{\left[1+C \cdot \mathrm{e}^{\frac{\sqrt{6 r}}{6} x}\right]^{2}}
$$

The results of the integration are given in Figure 4 where we have reported the comparison $F\left(x, t_{0}\right)$ and $G(x$, $t_{0}$ ) (namely the approximate and exact solutions vs. $x$ at fixed $t$ ) and the agreement is satisfactory.

The logistic behaviour of the solution (2.1) is shown in Figure 5 where we have reported the time counterpart, namely $F\left(x_{0}, t\right)$ and $G\left(x_{0}, t\right)$ of Figure 2 on linear scale otherwise difference are not evident.

In a more recent paper [10] an exact solution involving hyperbolic function has been given in the form

$$
S(x, t)=\frac{1}{2}\left[1+\tanh \left(-\frac{x}{4}+\frac{5}{8} t\right)\right]
$$

The comparison with the quasi exact form is shown in Figure 6 and the relevant agreement with $F(x, t)$ is significantly good.

Other examples could be discussed, but will not be reported here, the general consistency of our semi-analytical approach is confirmed at least for the simple form endowed in Equation (1.1). In the forthcoming section we will consider a comparison with a full numerical procedure along with some concluding remarks.

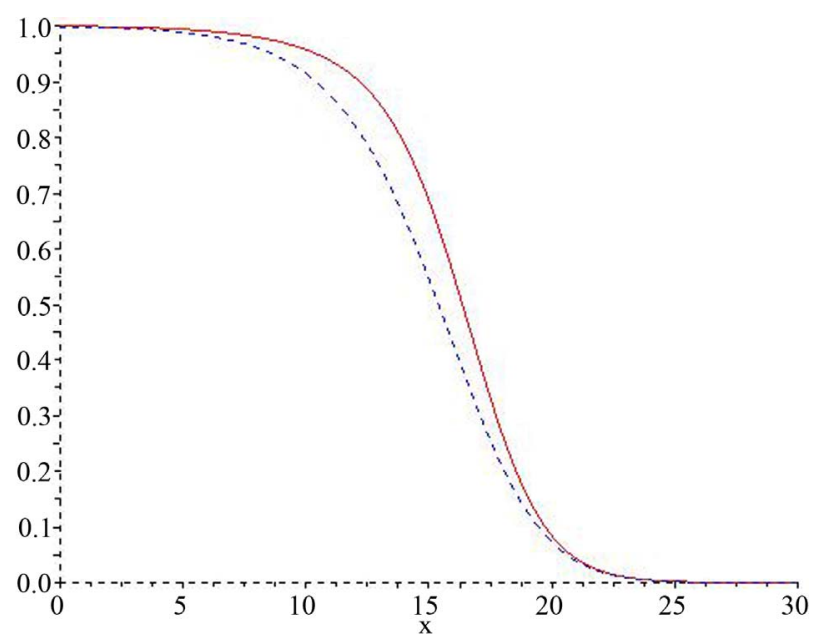

Figure 4. Exact solution (2.1) (dot), approximate solution (1.5) (continuous) for $t=10$, the agreement remains satisfactory at any time.

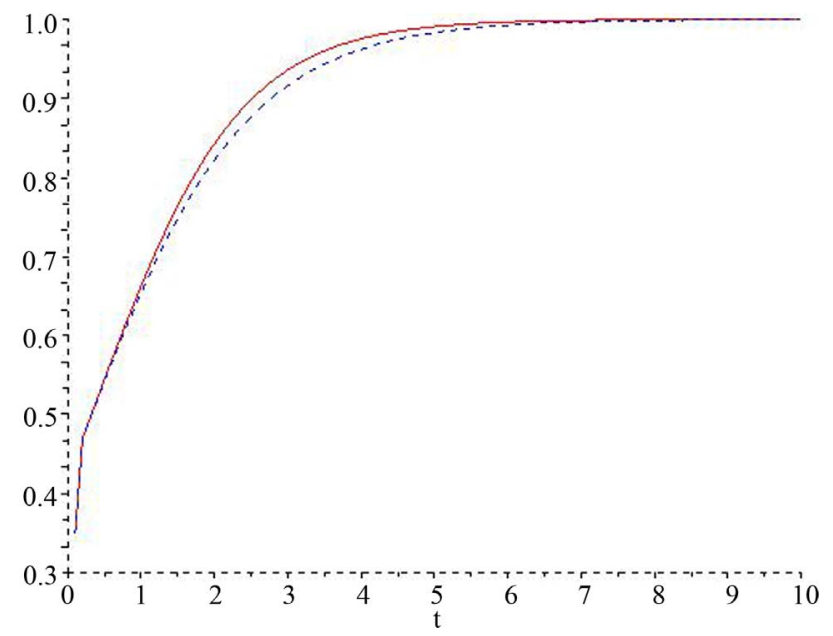

Figure 5. Same as Figure 2 for exact $G(0, t)$ (dot) and approximate $F(0, t)$ (continuous) solutions.

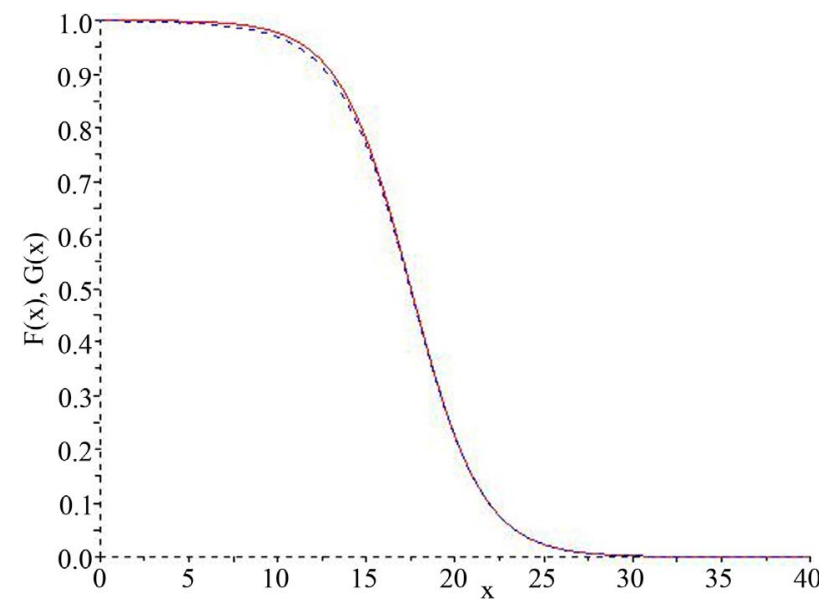

Figure 6. Exact solution (2.3) (dot), approximate solution (1.5) (continuous) for $t=7$, the solutions are not distinguishable at any time.

\section{Comparison with Numerical Solutions and Final Comments}

As already stressed, the exact solutions of the Fisher equation, used in the previous section as benchmarking of the validity of Equation (1.5), refer to specific cases of initial conditions. They are of limited usefulness in the application because they refer to conditions hardly applicable to problems concerning evolution of populations, genes or saturation mechanisms in laser Physics.

We will therefore further benchmark our solution with a series of numerical test using an ad hoc developed code.

The solution of Equation (1.1) will be afforded numerically by means of an iterative procedure employing a symmetric split decomposition [11-13]. We reconsider the Equation (1.1) in the form

$$
\partial_{t} F=D \partial_{x}^{2} F+\Lambda F,\left.F\right|_{t=0}=g(x)
$$


where $\Lambda$ is assumed to be a function of the spatial coordinate (as well as of time) but independent of the function $F$. The formal solution of our problem can accordingly be written as

$$
F(x, t)=\exp \left(t\left(D \partial_{x}^{2}+\Lambda\right)\right) g(x)
$$

Since the function $\Lambda$ is depending on the spatial coordinate we cannot use a naïve disentanglement of the exponential, a convenient solution based on a symmetric decomposition scheme is eventually provided by

$$
\mathrm{e}^{\delta\left[D \partial_{x}^{2}+\Lambda\right]} \cong \mathrm{e}^{\frac{\delta}{2} D \partial_{x}^{2}} \mathrm{e}^{\delta \Lambda} \mathrm{e}^{\frac{\delta}{2} D \partial_{x}^{2}}+o\left(\delta^{3}\right)
$$

Where $o\left(\delta^{3}\right)$ specifies the error of the procedure, which is associated (among other things discussed in the following) with the integration step $\delta$.

If we divide the integration interval in $N$ interval each one of size $\delta$, the solution of our problem (3.2) can be cast in the form

$$
F(x, t)=\left[\mathrm{e}^{\frac{\delta}{2} D \partial_{x}^{2}} \mathrm{e}^{\delta \Lambda} \mathrm{e}^{\frac{\delta}{2} D \partial_{x}^{2}}\right]^{N} g(x)
$$

which yields the following recursion

$$
\begin{aligned}
& F_{n}=\left[\mathrm{e}^{\frac{\delta}{2} D \partial_{x}^{2}} \mathrm{e}^{\delta \Lambda} \mathrm{e}^{\frac{\delta}{2} D \partial_{x}^{2}}\right] F_{n-1}, \\
& F_{0}=g(x)
\end{aligned}
$$

We can now go back to the Fisher equation and make the following substitution in Equation (3.5)

$$
\Lambda=r\left(1-\frac{F_{n-1}}{K}\right)
$$

Thus finally ending up with the "linearized solution"

$$
F_{n}=\mathrm{e}^{r \delta}\left[\mathrm{e}^{\frac{\delta}{2} D \partial_{x}^{2}} \mathrm{e}^{-\delta \frac{F_{n-1}}{K}} \mathrm{e}^{\frac{\delta}{2} D \partial_{x}^{2}}\right] F_{n-1}
$$

From the dynamical point of view we are left with a diffusive evolution problem counteracted by a spatially dependent growth rate. An example of comparison between numerical and solution with the previously outlined method and Equation (1.5) is given in Figure 7, where we have considered an initially Gaussian distribution ruled by a FE and the agreement can be considered fairly accurate. The numerical procedure we have developed is based on the symmetric split method, which is affected by an intrinsic error specified by [11-13]

$$
\mathrm{e}^{\delta(A+B)} \cong \mathrm{e}^{\frac{1}{2} \delta A} \mathrm{e}^{\delta B} \mathrm{e}^{\frac{1}{2} \delta A}+\frac{\delta^{3}}{24}[A,[A, B]]
$$

The error is accordingly specified by the discretization step and by the commutator involving the operators $A, B$, namely

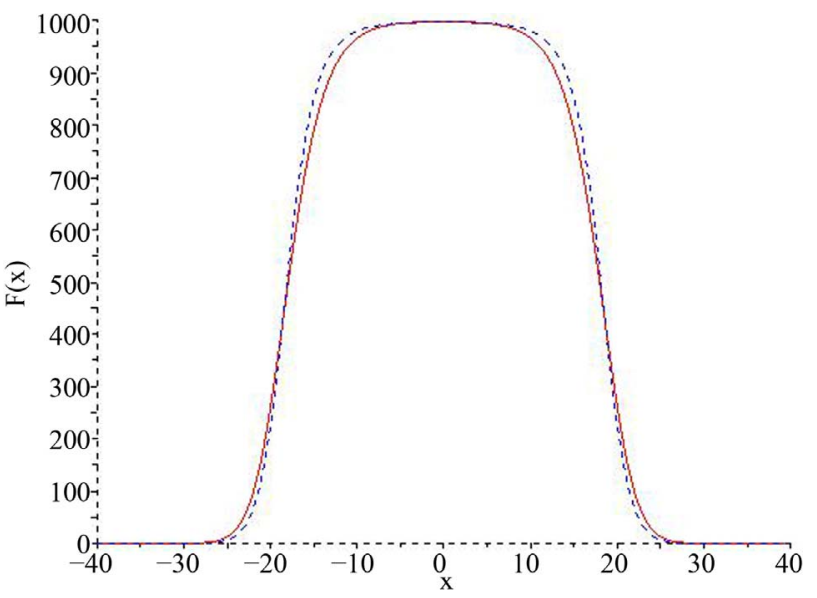

Figure 7. Comparison between numerical (dot) and semianalytical solution (dash) same parameters of Figure 1.

$$
\begin{aligned}
& {[A,[A, B]] \propto D^{2} r\left[\partial_{x}^{2},\left[\partial_{x}^{2}, F_{n-1}\right]\right]} \\
& C_{n-1}=\left[\partial_{x}^{2},\left[\partial_{x}^{2}, F_{n-1}\right]\right]
\end{aligned}
$$

Abrupt variations of the function itself at different evolution steps may provide a large error even for small $\delta$, furthermore a large number of iterations may determine an accumulation of error which can be computed from

$$
E \cong \frac{\delta^{3}}{24} \sum_{r=1}^{N} C_{n-1} F_{n-1}
$$

The integrals regarding the Weierstrass transform are explicitly derived using a Simpson scheme. The homemade code even though accurate is not optimized and we have used repeatedly the combinations of two numerical errors due to the split operator and to the Simpson integration method. To make sure of the comparison we have attempted a second numerical procedure, based on FFT algorithm implemented in Mathematica.

A 3-D perspective of the numerical integration is given in Figure 8, which clearly displays the dynamical features discussed in the previous parts of the paper.

In this case too the comparison between analytical and numerical solutions yields a satisfactory agreement.

The solution we have proposed, albeit naïve, seems to be rather efficient in solving the problem of providing the evolution of a population governed by the Fisher equation. Our solution is based on the requirement that it should provide the logistic and diffusive behavior, implicit in the assumption underlying its original derivation in [1].

It should be not surprising that this family of equations can be solved in a simple form since the logistic equation, even though non-linear can be reduced to a linear form by an appropriate change of variable and more in general of Burger type equations, which can be reduced to a heat 


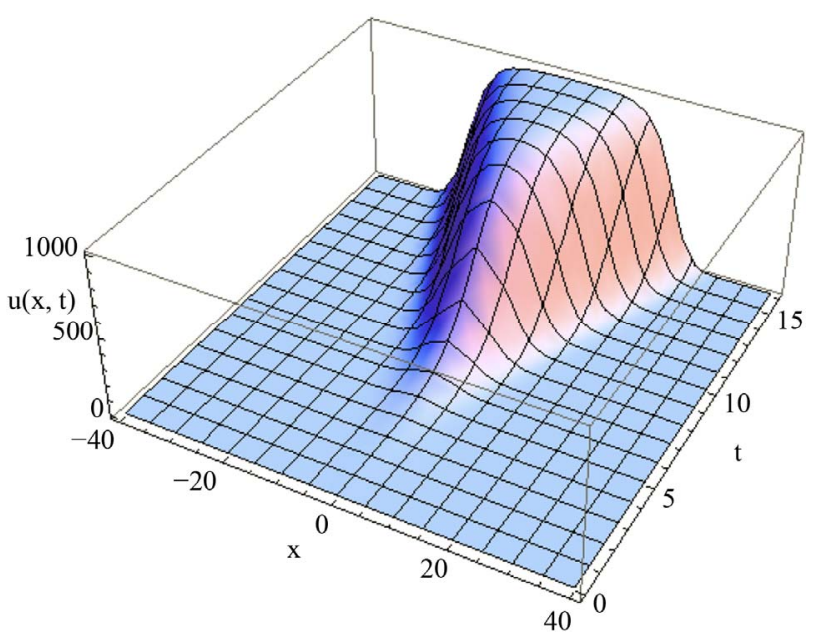

Figure 8. Three dimensional evolution $(t, x)$ of the Fisher distribution.

equation by means of a Hopf-Cole transformation [14].

The following example can be useful to support the previous argument, by considering the differential equation

$$
\begin{aligned}
& \partial_{t} S=v \partial_{x} S+r\left(1-\frac{S}{K}\right) S, \\
& S(x, 0)=g(x)
\end{aligned}
$$

In this case we are not dealing with a diffusive Fisher equation but with a simpler form involving a logistic saturation and a coordinate translation ${ }^{3}$. According to the same arguments invoked for the FE, we "guess" that the solution of Equation (3.11) writes ${ }^{4}$

$$
S(x, t)=\frac{g(x+v t) \mathrm{e}^{r t}}{1+\frac{1}{K}\left(\mathrm{e}^{r t}-1\right) g(x+v t)}
$$

namely the composition of a translation and of a logistic. The above solution can be obtained in rigorous terms after setting $^{5}$

$$
F(x, t)=(u(x, t))^{-1}
$$

The transformation (3.13) eliminates the non-linearity in Equation (3.11) and this allows the solution in exact form (see [3] for further details).

Regarding the Fisher equation, the presence of the second derivative does not allow the elimination of the

${ }^{3}$ INote that $\exp \left(v t \partial_{x}\right) g(x)=g(x+v t)$

${ }^{4}$ Regarding the FE solution we have also tried the form

$$
F(x, t)=[\exp (r t) /(1+(1 / K)(\exp (r t)-1) \tilde{F}(x, t))] \tilde{F}(x, t)
$$

but we did not find any significant differences with respect to the case of Equation (1.5).

${ }^{5}$ It can indeed be written as

$$
\left(\partial_{t}-v \partial_{x}\right) u=-r(u-1 / K)
$$

non- linearity, the corresponding equation reads indeed

$$
\partial_{t} u=D \partial_{x}^{2} u-r\left(u-K^{-1}\right)-2 D \frac{\left(\partial_{x} u\right)^{2}}{u}
$$

which contains a non-linearity which can be neglected whenever

$$
\left|\frac{\left(\partial_{x} u\right)^{2}}{u}\right|<<\left|\partial_{x}^{2} u\right|
$$

which is not verified for large values of $F(x, t)$, namely in the region in which the saturation occurs.

In a forthcoming investigation we will extend the analysis to multi-dimensional (2-D, 3-D) configurations and discuss specific examples of applications.

\section{Acknowledgements}

The authors express their sincere appreciation to Prof. F. Pacella for the kind interest and for a critical reading of the manuscript.

\section{REFERENCES}

[1] R. A. Fisher, "The Wave of Advance of Advantageous Genes," Annals of Genetics, Vol. 7, No. 4, 1937, p. 353.

[2] A. Kolmogorov, I. Petrovskii and N. Piscounov, "Etude de L'équation de la Diffusion Avec Croissance de la Quantité de Matière et Son Application a un Problem Biologique," In: V. M. Tikhomirov, Ed., Selected Works of A. N. Kolmogorov I, Kluwer, Dordrecht, 1991, p. 248.

[3] B. H. Gilding and R. Kersner, "Travelling Waves in Nonlinear Diffusion Convection Reaction," Birkhäuser, Basel, 2004. doi:10.1007/978-3-0348-7964-4

[4] J. Vandermeer, "How Populations Grow: The Exponential and Logistic Equations," Nature Education Knowledge, Vol. 1, No. 8, 2010, p. 1.

[5] D. Babusci, G. Dattoli and M. Delfranco, "Lectures on Mathematical Methods for Physics," RT/2010/58/ENEA. http://www.frascati.enea.it/biblioteca

[6] E. E. Holmes, M. A. Lewis, J. A. Banks and R. R. Veit, "Partial Differential Equations in Ecology: Spatial Interactions and Population Dynamics," Ecology, Vol. 75, No. 1, 1994, pp. 17-29. doi:10.2307/1939378

[7] M. J. Ablowitz and A. Zeppetella, "Explicit Solutions of Fisher's Equation for a Special Wave Speed," Bulletin of Mathematical Biology, Vol. 41, No. 6, 1979, pp. 835-840.

[8] N. A. Kudryashov, "On Exact Solutions of Families of Fisher Equations," Theoretical and Mathematical Physics, Vol. 94, No. 2, 1993, pp. 211-218.

[9] V. G. Danilov, V. P. Maslov and K. A. Volosov, "Mathematical Modelling of Heat and Mass Transfer Processes," Kluwer, Dordrecht, 1995.

[10] E. D. Kocacoban, A. B. Koc, A. Kurnaz and Y. Keskin, "A Better Approximation to the Solution of Burger Fisher Equation," Proceedings of the World Congress on Engineering 2011, London, 6-8 July 2011. 
[11] G. Strang, "On the Construction and Comparison of Difference Schemes," SIAM Journal on Nu-merical Analysis, Vol. 5, No. 3, 1968, pp. 506-517.

[12] G. Dattoli, P. L. Ottaviani, A. Torre and L. Vazquez, "Evolution Operator Equations: Integration with Algebraic and Finite Difference Methods. Application to Physical Problems in Classical and Quantum Mechanics and Quantum Field Theory," La Rivista Del NuovoCimento, Vol. 20, No. 2, 1997, pp. 3-133.
[13] S. Blanes, F. Casas and A. Murua, "Symplectic Splitting Operator Methods Tailored for the Time-Dependent Schrodinger Equation," Journal of Chemical Physics, Vol. 124, 2006, Article ID: 234105. doi:10.1137/0705041

[14] L. Giuggioli and V. M. Kenkre, "Analytic Solutions of a Nonlinear Convective Equation in Population Dynamics," Physica D: Nonlinear Phenomena, Vol. 183, No. 3-4, 2003, pp. 245-259. doi:10.1016/S0167-2789(03)00176-3 